\title{
Mode dynamics and thermal lens effects of thin-disk lasers
}

\author{
J. Mende*, J. Speiser, G. Spindler, W. L. Bohn, A. Giesen \\ German Aerospace Center, Institute of Technical Physics, \\ Pfaffenwaldring 38 - 40, D-70569 Stuttgart, Germany
}

\begin{abstract}
In principle, the thin-disk laser concept opens the possibility to demonstrate high power, high efficiency and good beam quality, simultaneously. For this purpose, a very homogeneous pump power distribution on the disk is necessary as well as very low phase distortions of the disk itself.

Spatial mode structure and thermal lens effects in an Yb:YAG thin-disk laser have been investigated as function of the pump power in linear and folded resonators. Whereas thermal lens is shown to be very weak due to the thin disk geometry, a strong correlation of the laser mode with respect to the power density distribution of the pump radiation is exhibited. The experimental results are compared with numerical simulations of the field distribution within the resonator as well as in the far field demonstrating the excellent homogeneity of the disk as laser active medium.
\end{abstract}

The results will be used for scaling the fundamental mode power into the multi-kW-regime.

Keywords: thin-disk laser, spatial mode structure, mode dynamics, simulation of field distribution, power scalability

\section{INTRODUCTION}

Due to thermal effects within the laser active medium, the brightness of conventional solid state lasers is limited. Therefore, in the past years many investigations to enhance the brightness of high power solid state lasers were made.

The thin-disk laser concept offers the possibility to realize lasers with high efficiency and good beam quality. The minimization of the thermal lens and thermal aberrations allows the power scalability of this type of lasers even in fundamental mode. Besides the optimization of the thin disk itself, this requires also a sophisticated resonator design.

Here we report on the investigations of the mode structures and dynamics in linear and folded resonators as well as the comparison of the results with numerical simulations. With an Yb:YAG thin-disk laser, an output power of $360 \mathrm{~W}$ in fundamental mode operation has been achieved.

\section{THE THIN-DISK LASER CONCEPT}

The main advantage of the thin-disk laser is the highly efficient cooling of the laser medium due to the advantageous ratio of surface to volume. The cooling geometry leads to a quasi one-dimensional temperature profile. Because of the collinearity of the temperature gradient and the laser beam propagation, thermal lens effects are minimized (Fig. 1). The highest published output power of an $1030 \mathrm{~nm} \mathrm{Yb:YAG} \mathrm{single} \mathrm{disk} \mathrm{laser} \mathrm{is} 5.3 \mathrm{~kW}$ in multi mode operation ${ }^{1}$. Fundamental mode operation with an output power of $225 \mathrm{~W}$ has been reported ${ }^{2}$.

*jens.mende@dlr.de; phone ++49 711 / 6862 - 229; fax -715 ; www.dlr.de 


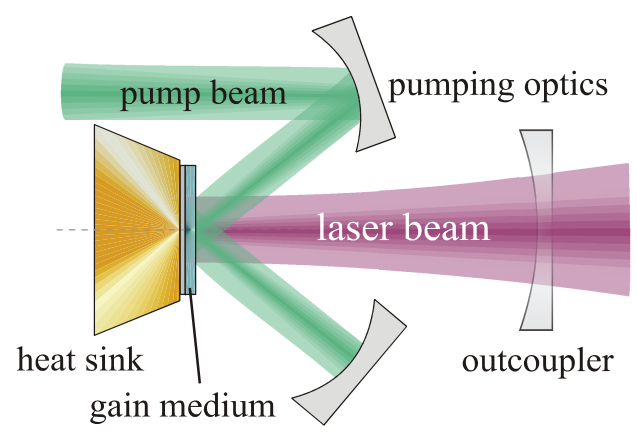

Fig. 1: Principle of the thin-disk laser: the gain medium consists of a thin crystal disk which is mounted on a heat sink. The pump radiation is quasi-longitudinally focused on the disk with pump optics. The rear side of the disk is HR coated for the pump wavelength as well as the laser wavelength. Due to the multi-pass of the pump beam through the disk, a high energy deposition in the laser medium is achieved. The thin disk itself is part of the resonator as a HR mirror.

\section{CHARACTERIZATION OF THIN-DISK LASER MODULES}

\subsection{Characterization of the pump radiation}

To characterize the homogeneity of the pump power, the fluorescence intensity distribution on the disk has been imaged on a camera. The detected radiation consists of scattered pump radiation and fluorescence radiation (Fig. 2, Fig. 3). The fluorescence intensity is approximately proportional to the spatial distribution of the inversion density and hence to the absorbed pump power.
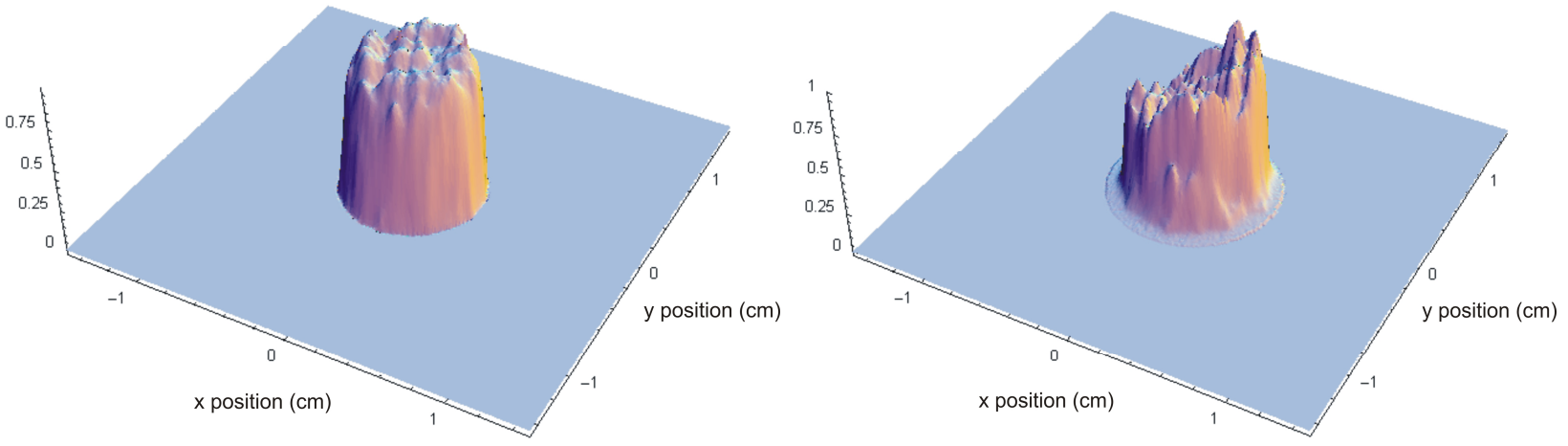

Fig. 2: Normalized fluorescence intensity distribution on the disk. Left: using 16 pump passes; right: two pump passes. 

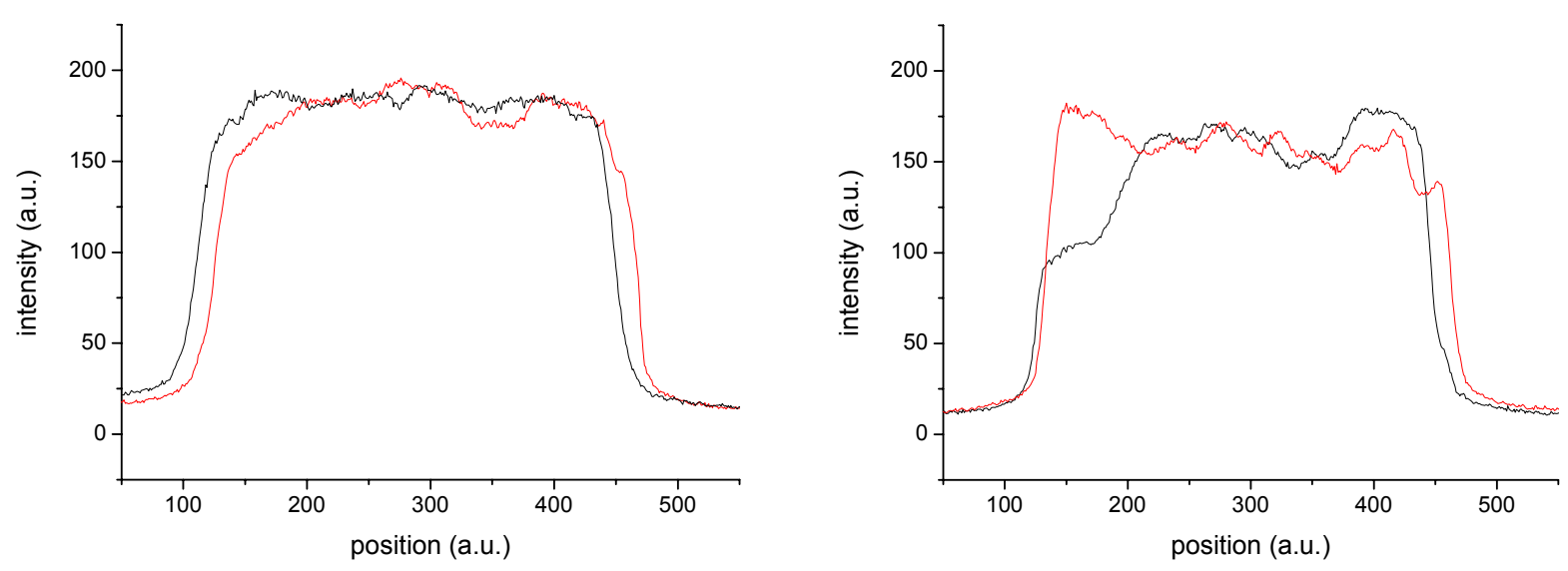

Fig. 3: Fluorescence intensity profiles on the disk in two orthogonal directions. Left: using 16 pump passes; right: two pump passes.

The pump radiation enters the thin disk and is reflected at its rear side and passes through the disk a second time. Due to the repeated pump radiation passes, caused by the pumping optics, the resulting pump profile is a superposition of 16 single profiles which are rotated around the laser axis by re-imaging on the disk. This reduces the inhomogeneity in the pump profile. The inhomogeneity is more significant, if one uses only two pump passes.

\subsection{Characterization of the laser active medium}

Due to the disk geometry, thermal lens effects in thin-disk lasers are very weak. However, at high power, there are still considerable variations of the refraction power of the gain medium. To measure this variation, a disk was irradiated with an collimated beam of an Nd:YAG laser and the position of the beam waist of the reflected beam was mapped.

The investigated disk shows a concave curvature and the focal length $f_{\mathrm{g}}$ increases linearly with pump power (Fig 4). The consideration of this dependency is necessary for the design of the resonator, especially with serial or parallel coupled thin-disk modules and with respect to dynamic stability of the resonator.
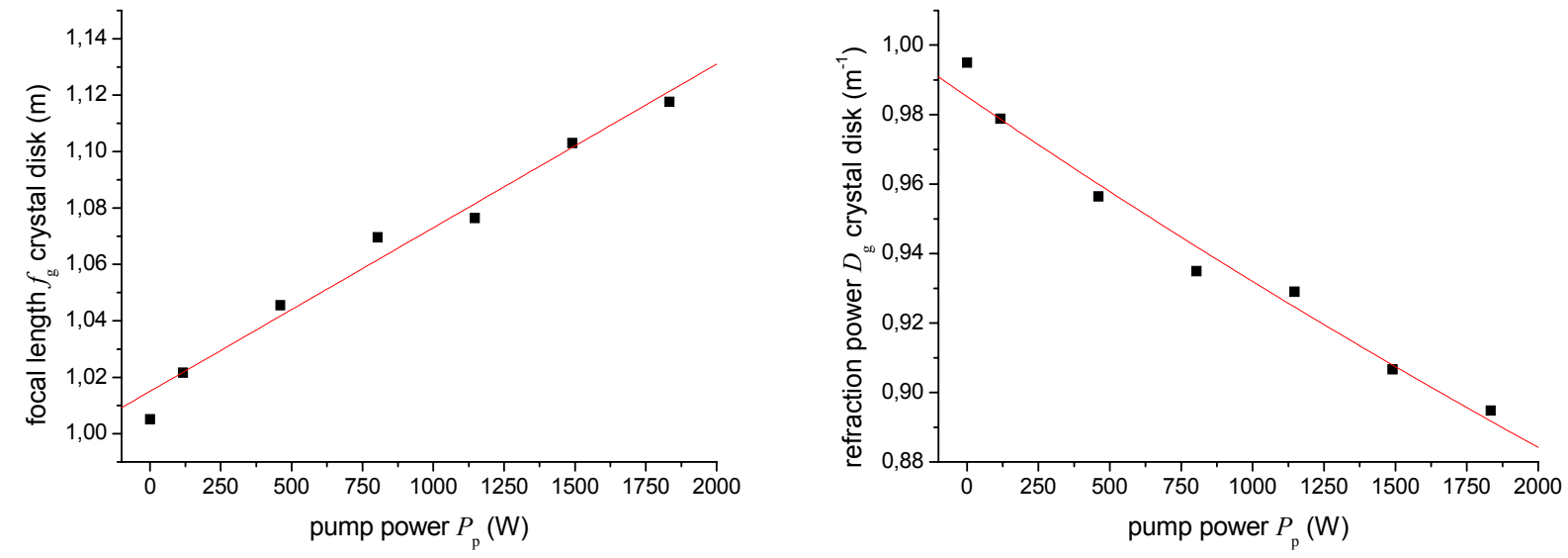

Fig 4: Focal length $f_{\mathrm{g}}(\mathrm{left})$ and refraction power $D_{\mathrm{g}}$ (right) of the investigated disk during laser operation versus pump power $P_{\mathrm{p}}$. 


\subsection{Characterization of a compact linear resonator}

To characterize the output power of a laser with a single thin-disk module an optimized energy conversion of pump power into laser emission was pursued. To accomplish this, a short linear resonator was investigated (Fig. 5).

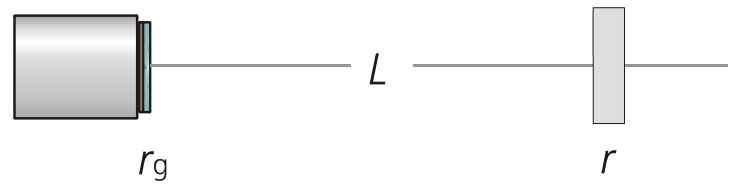

Fig. 5: Linear resonator with a single thin-disk module $\left(L=0.38 \mathrm{~m} ; r=\propto ; r_{\mathrm{g}} \in[2 \mathrm{~m} ; 2,2 \mathrm{~m}]\right)$.

This linear resonator allows high order multi-mode operation, resulting in a poor beam quality. Due to the very small fundamental mode radius $\left(w_{0 \mathrm{~g}}=0.5 \mathrm{~mm}\right)$ compared to the pump spot radius $\left(r_{\mathrm{p}}=4.15 \mathrm{~mm}\right)$, the resulting laser mode shows an optimal overlap with the pump spot. Fig. 6 shows the output power $P_{\text {out }}$ and the optical efficiency $\eta$ of this resonator.
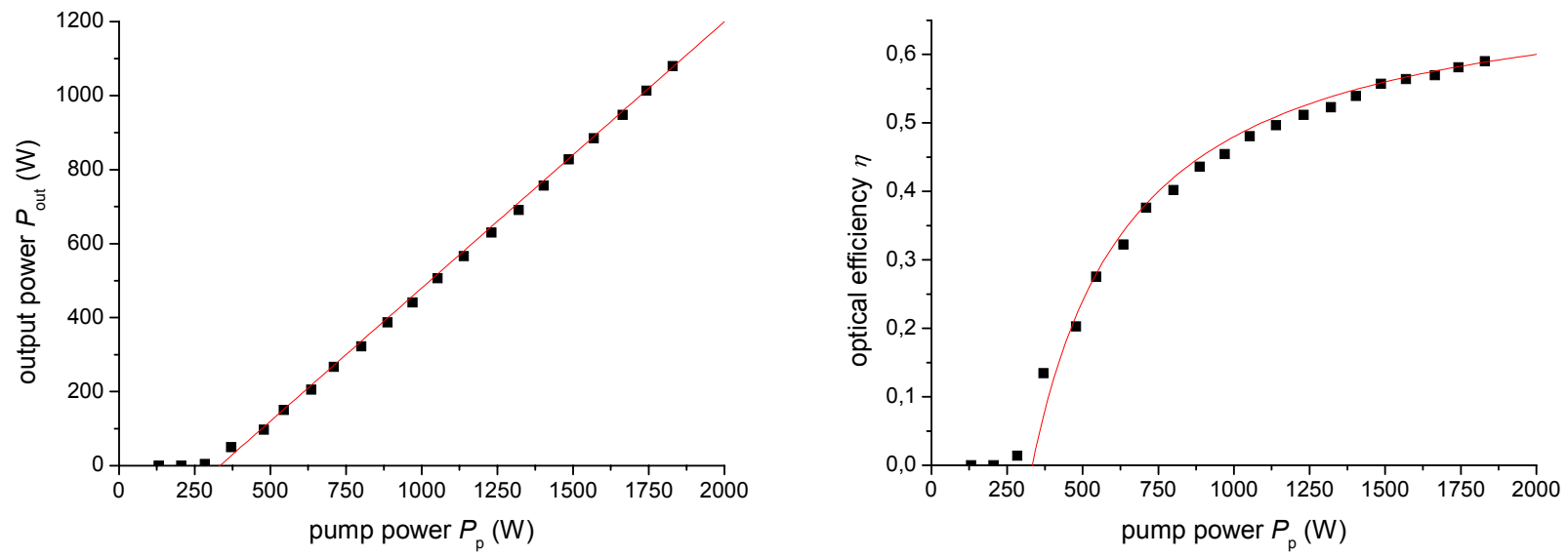

Fig. 6: Output power $P_{\text {out }}$ and optical efficiency $\eta$ of the linear resonator as function of the pump power $P_{\mathrm{p}}$.

A maximum output power of $1.08 \mathrm{~kW}$ in multi-mode operation has been achieved with an efficiency of $59 \%$ and an outcoupling transmission of $3 \%$. Because of a typical pump efficiency of about $90 \%$ and a Stokes efficiency of $91 \%$, the slope efficiency of $71 \%$ indicates that the losses are very small.

Besides the power characterization, beam profiles of the linear resonator were recorded as function of the output power (Fig. 7). Increasing the pump power, the linear resonator oscillates in higher order modes showing rectangular symmetry. 


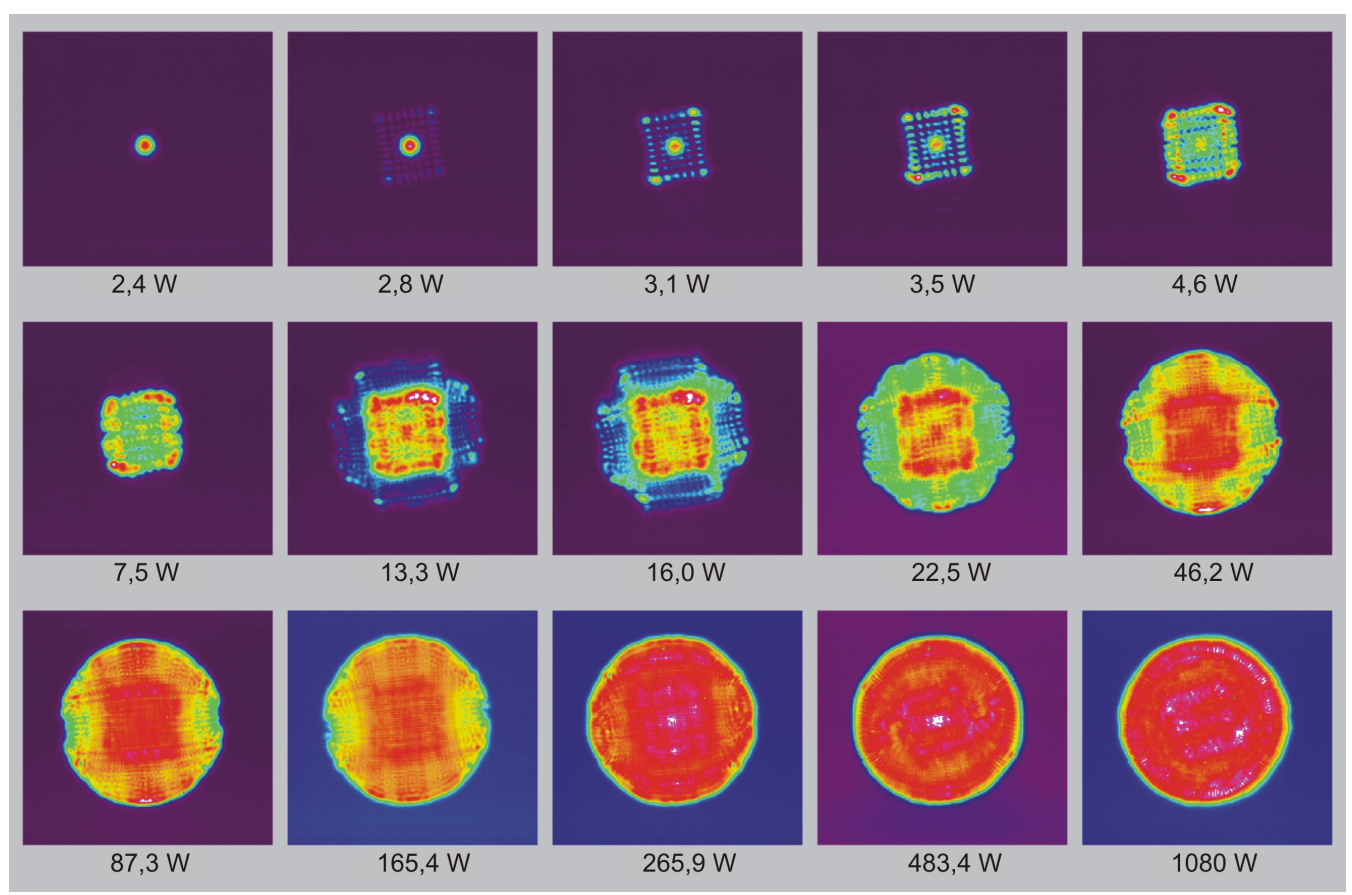

Fig. 7: Beam profiles of the linear resonator as function of the output power $P_{\text {out }}$.

\subsection{Design and characterization of $\mathrm{V}$-shaped resonators}

The beam quality and the efficiency of several folded resonators (V-shaped resonators) have been investigated (Fig. 8).

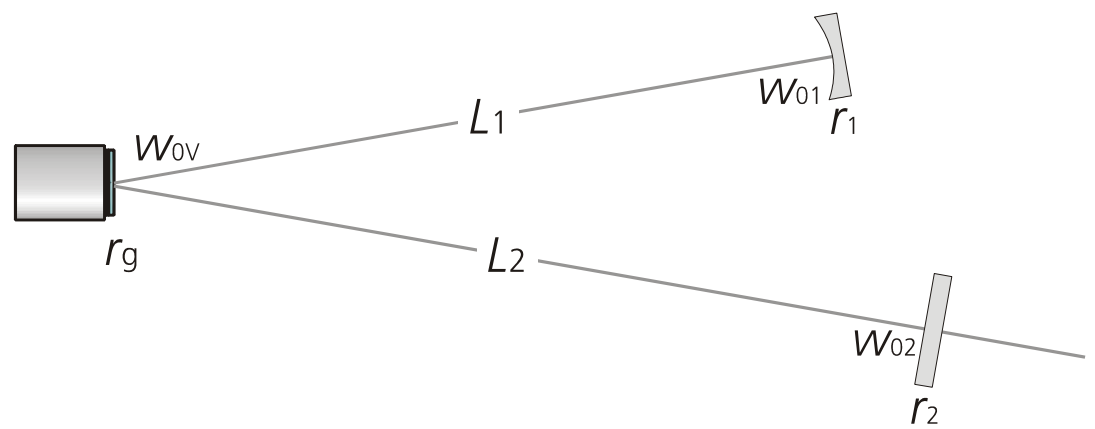

Fig. 8: V-shaped resonator with a thin-disk module as deflecting mirror $\left(r_{1,2, \mathrm{~g}}=\right.$ curvature radii of HR mirror, outcoupler and disk, respectively; $L_{1,2}=$ distances disk to mirrors; $w_{01,02,0 \mathrm{~V}}=$ mode radii on mirrors and disk, respectively).

The pump spot on the disk acts just like a soft mode aperture. A significant beam quality improvement can be achieved by adaptation of the fundamental mode radius to the pump spot radius via appropriate choice of the mirror curvature radii and of the resonator length. Thus, only the fundamental mode is amplified and higher order modes are suppressed $^{3,4}$.

According to our numerical simulations and our experimental results, there is an optimized overlap between the fundamental mode radius $w_{0 \mathrm{~V}}$ on the disk and the pump spot radius $r_{\mathrm{p}}$ for the condition $w_{0 \mathrm{~V}} \in\left[0,6 r_{\mathrm{p}} ; 0,8 r_{\mathrm{p}}\right]$. These values depend on the outcoupling transmission and other losses. 
The investigated V-shaped resonators were dynamically stable, so the laser mode radius varies only slightly if the focal length of the disk changes due to thermal lens effects. Thus, the beam quality is nearly constant with increasing pump power.

Not depending on the exact resonator configuration, corresponding to the theory of stable resonators ${ }^{4}$, the stability range of a resonator with a thermal lens with the focal length $f_{\mathrm{g}}$ is given by $\Delta\left(\frac{1}{f_{\mathrm{g}}}\right)=\frac{2 \lambda}{\pi w_{0 V}{ }^{2}}$.

In our case we have $w_{0 \mathrm{~V}}=0.65 r_{\mathrm{p}} \approx 2.7 \mathrm{~mm}$, so the stability range is $\Delta\left(\frac{1}{f_{\mathrm{g}}}\right)=0.097 \mathrm{~m}^{-1}$.

At the limits of the stability range the fundamental mode radius as a function of the thermal lens increases rapidly. Therefore, about one half of the stability range is usable. The change of the thermal lens of our disks is about $0.05 \mathrm{~m}^{-1} \mathrm{~kW}^{-1}$, so a stable fundamental mode operation should be possible for a pump power range of $1 \mathrm{~kW}$.

In the following the experimental results of two investigated dynamically stable V-shaped resonators - one with multimode emission and one with fundamental mode emission - are discussed. The resonator data are listed in the table below:

\begin{tabular}{|lll|}
\hline & $\begin{array}{l}\text { multi mode } \\
\text { V-shaped resonator }\end{array}$ & $\begin{array}{l}\text { fundamental mode } \\
\text { V-shaped resonator }\end{array}$ \\
$r_{1}[\mathrm{~m}]$ & 1.26 & 0.5 \\
$r_{2}[\mathrm{~m}]$ & $\propto$ & $\propto$ \\
$r_{\mathrm{g}}[\mathrm{m}]$ & $\in[2.0 \mathrm{~m}, 2.2 \mathrm{~m}]$ & $\in[2.0 \mathrm{~m}, 2.2 \mathrm{~m}]$ \\
$L_{1}[\mathrm{~m}]$ & 1.70 & 2.255 \\
$L_{2}[\mathrm{~m}]$ & 1.63 & 2.158 \\
$w_{0 \mathrm{~V}} / r_{\mathrm{p}}$ & 0.25 & 0.65 \\
\hline
\end{tabular}

To characterize the laser emission of these resonators, the output power, the optical efficiencies and the beam profiles as function of the output power were measured.

Multi-mode operation
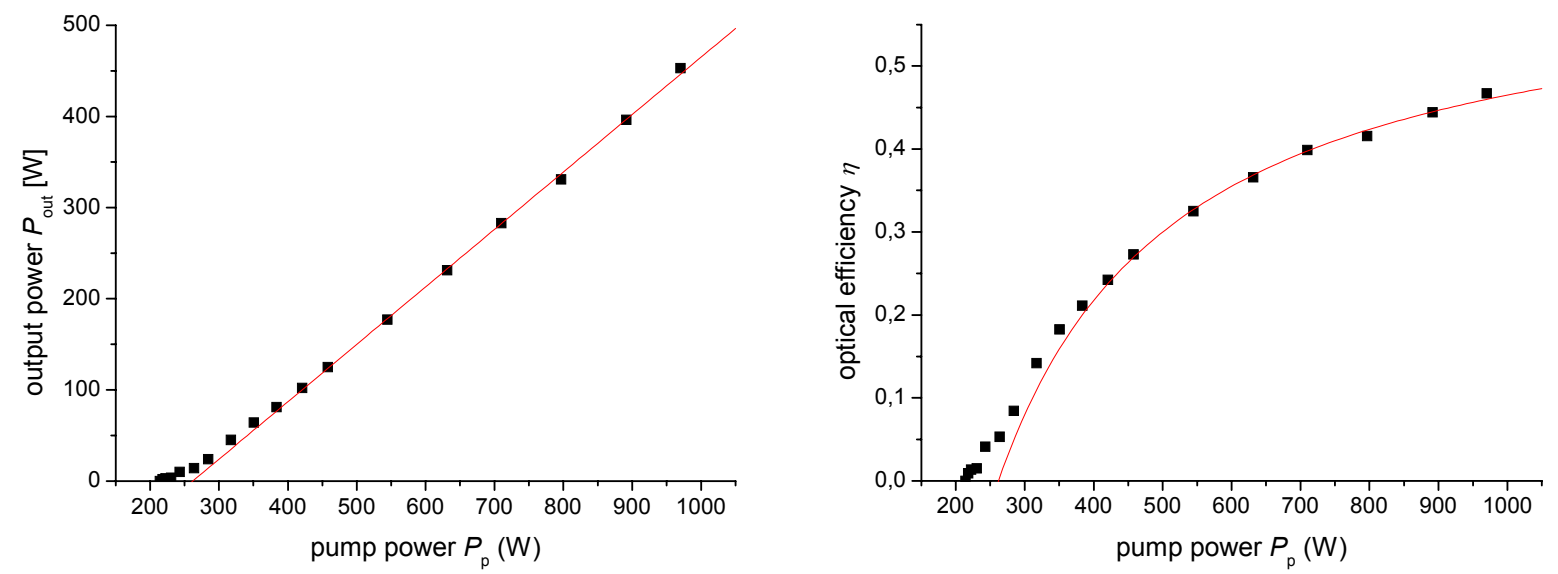

Fig. 9: Output power $P_{\text {out }}$ and optical efficiency $\eta$ of the multi mode V-shaped resonator as function of the pump power $P_{\mathrm{p}}$. 


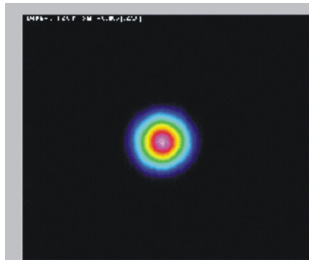

$<0,5 \mathrm{~W}$

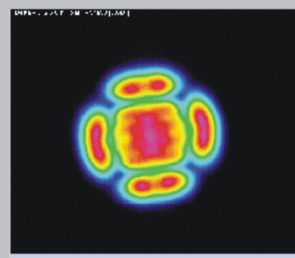

$2,0 \mathrm{~W}$

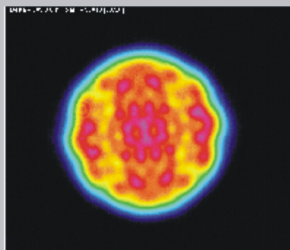

$10 \mathrm{~W}$

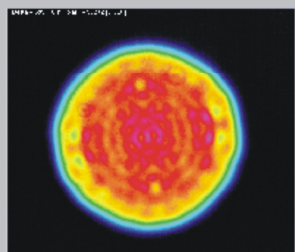

$60 \mathrm{~W}$

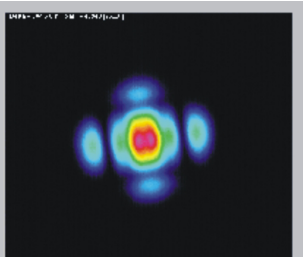

$<1,0 \mathrm{~W}$

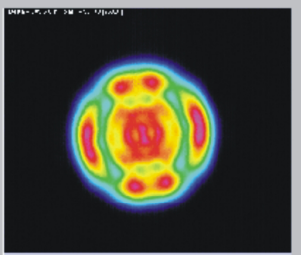

$2,5 \mathrm{~W}$

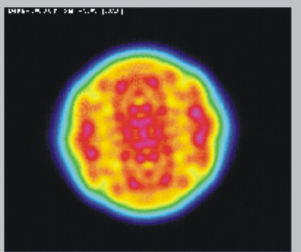

$14 \mathrm{~W}$

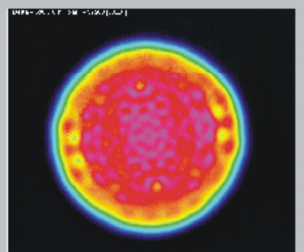

$90 \mathrm{~W}$

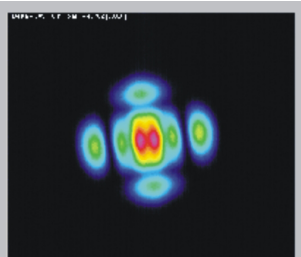

$1,0 \mathrm{~W}$

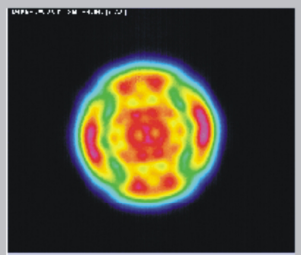

$3,0 \mathrm{~W}$

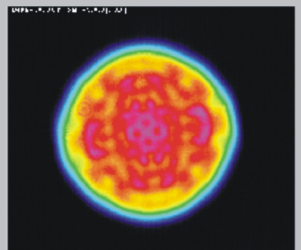

$24 \mathrm{~W}$

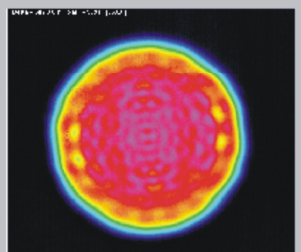

135 W

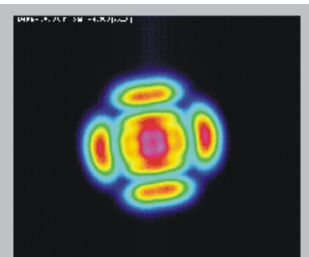

$1,5 \mathrm{~W}$

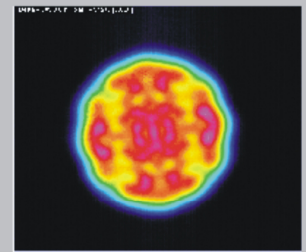

$7,0 \mathrm{~W}$

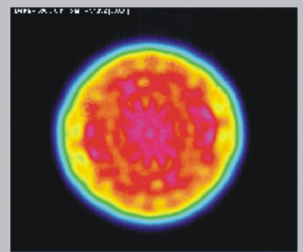

$40 \mathrm{~W}$

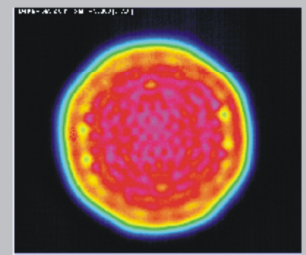

260 W

Fig. 10: Variation of the beam profile of the multi-mode V-shaped resonator with increasing output power $P_{\text {out }}$.

In the multi-mode $\mathrm{V}$-shaped resonator, higher order modes oscillate with increasing pump power similar to the results with the linear resonator. The beam profiles also show an influence of the pump power intensity distribution on the mode structure.

Numerical simulations of the resonator field distribution were done in this context, considering the real pump profile. Fig. 11 shows two calculated beam profiles near the laser threshold and at low output power qualitatively matching the experimental results in Fig. 10. 

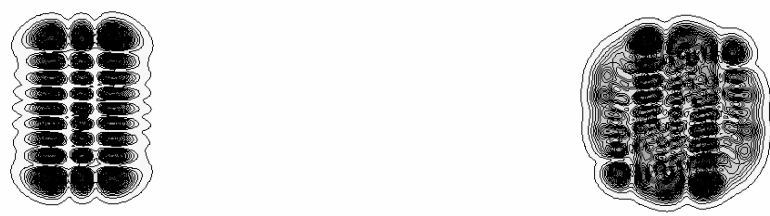

Fig. 11: Numerical simulations of the field distribution in the multi-mode V-shaped resonator near the threshold (left) and with an output power $P_{\text {out }}=4 \mathrm{~W}$ (right).

The measured beam profile at low output power could be explained as an oscillation between two perpendicularly orientated rectangular resonator modes. Polarization selective recordings of the beam profile were performed (

Fig. 12) and intensity measurements in the outer intensity maxima of the two modes in each polarization direction (Fig. 13) were made. The frequency of the intensity fluctuation is $135 \mathrm{MHz}$. This is equal to three times the axial mode spacing $(45 \mathrm{MHz})$ of the resonator.
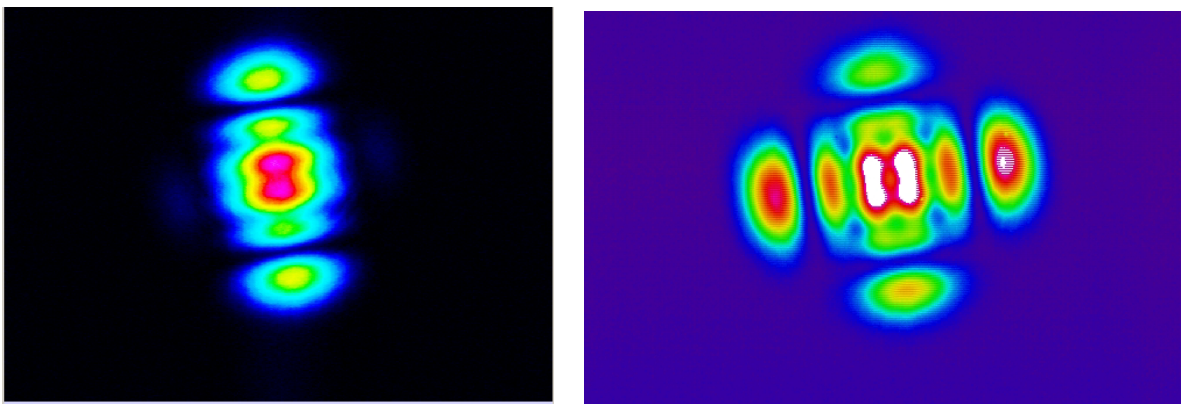

Fig. 12: Polarization selective beam profile measurements at low output power performed with a thin film polarizer. Left: p-polarization, right: s-polarization.

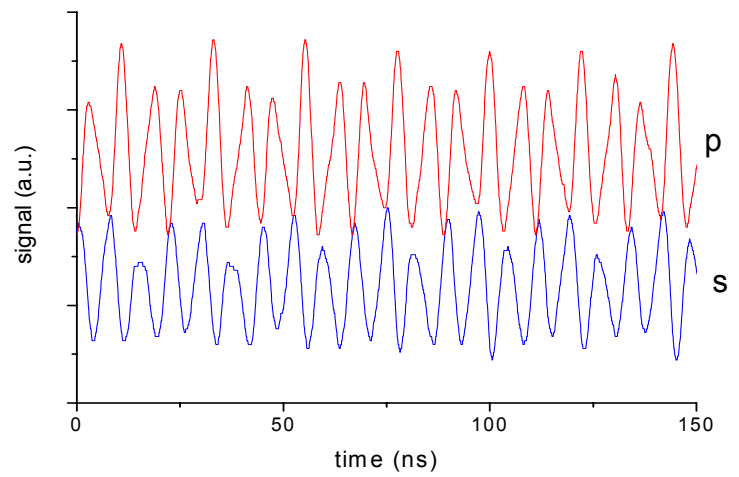

Fig. 13: Intensity fluctuations of the laser modes in p- and s-polarization with a frequency of $135 \mathrm{MHz}$. 


\section{Fundamental mode operation}

Adapting the fundamental mode radius on the disk to the pump spot radius, higher order modes could be suppressed successfully. An output power of $342 \mathrm{~W}$ with an efficiency of $32 \%$ in fundamental mode operation could be achieved (Fig. 14).
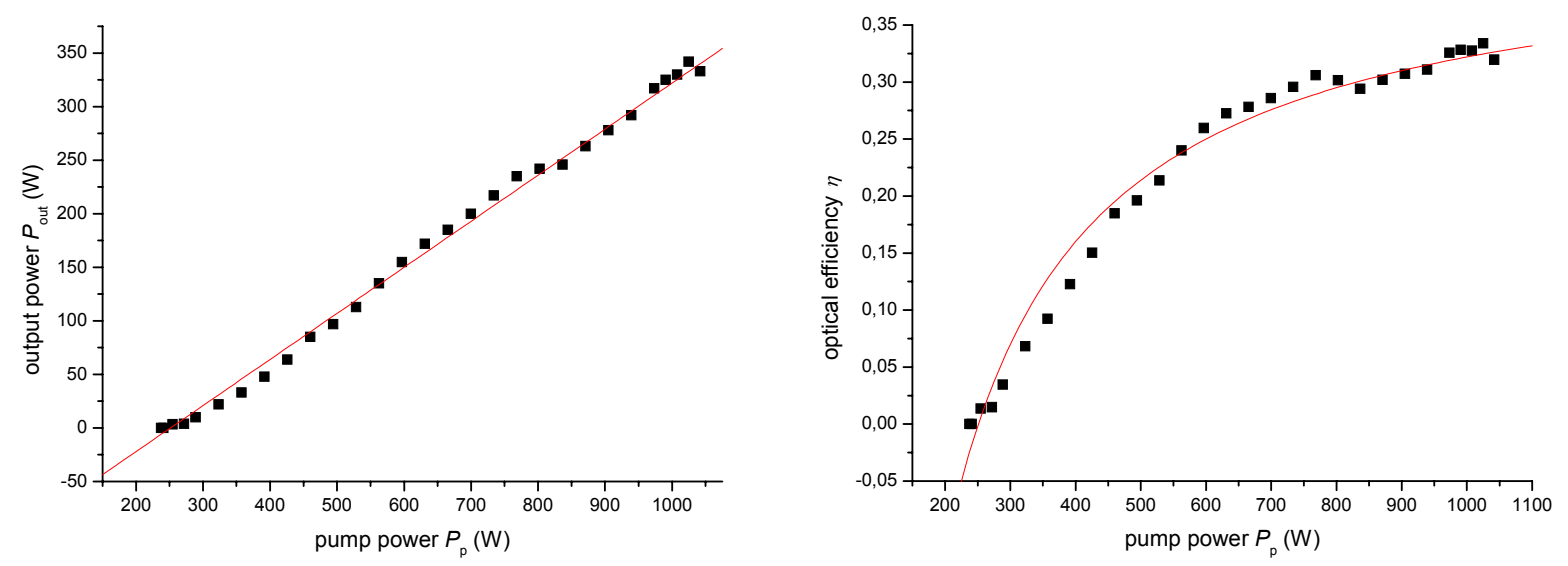

Fig. 14: Output power $P_{\text {out }}$ and optical efficiency $\eta$ of the dynamical stable fundamental mode V-shaped resonator as function of the pump power $P_{\mathrm{p}}$

The beam profiles in Fig. 15 and Fig. 16 show the experimental results and the numerical simulations of the fundamental mode V-shaped resonator.
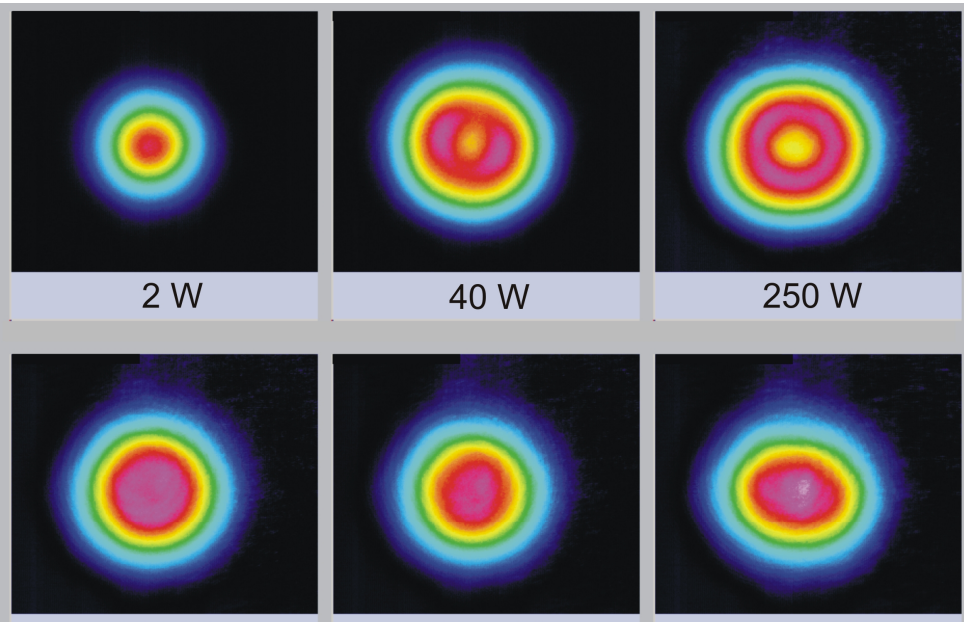

$266 \mathrm{~W}$

$290 \mathrm{~W}$

$250 \mathrm{~W}$

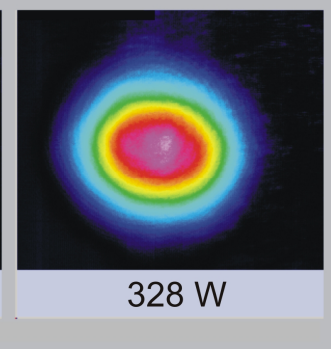

Fig. 15: Variation of the beam profile of the fundamental mode V-shaped resonator with increasing output power $P_{\text {out }}$.

Due to the resonator design the fundamental mode radius passes a minimum, when the radius of curvature of the disk grows with increasing pump power. In the range of the minimum radius, first order modes TEM $_{01}$ and TEM 10 were amplified as well. Further increase of pump power leads to a larger fundamental mode radius again, so the laser emission is back in fundamental mode $\mathrm{TEM}_{00}$ (Fig. 17). The inhomogeneity of the pump profile causes a reduced efficiency in the fundamental mode operation. Numerical simulations qualitatively yield the same results as the experiments (Fig. 16). 


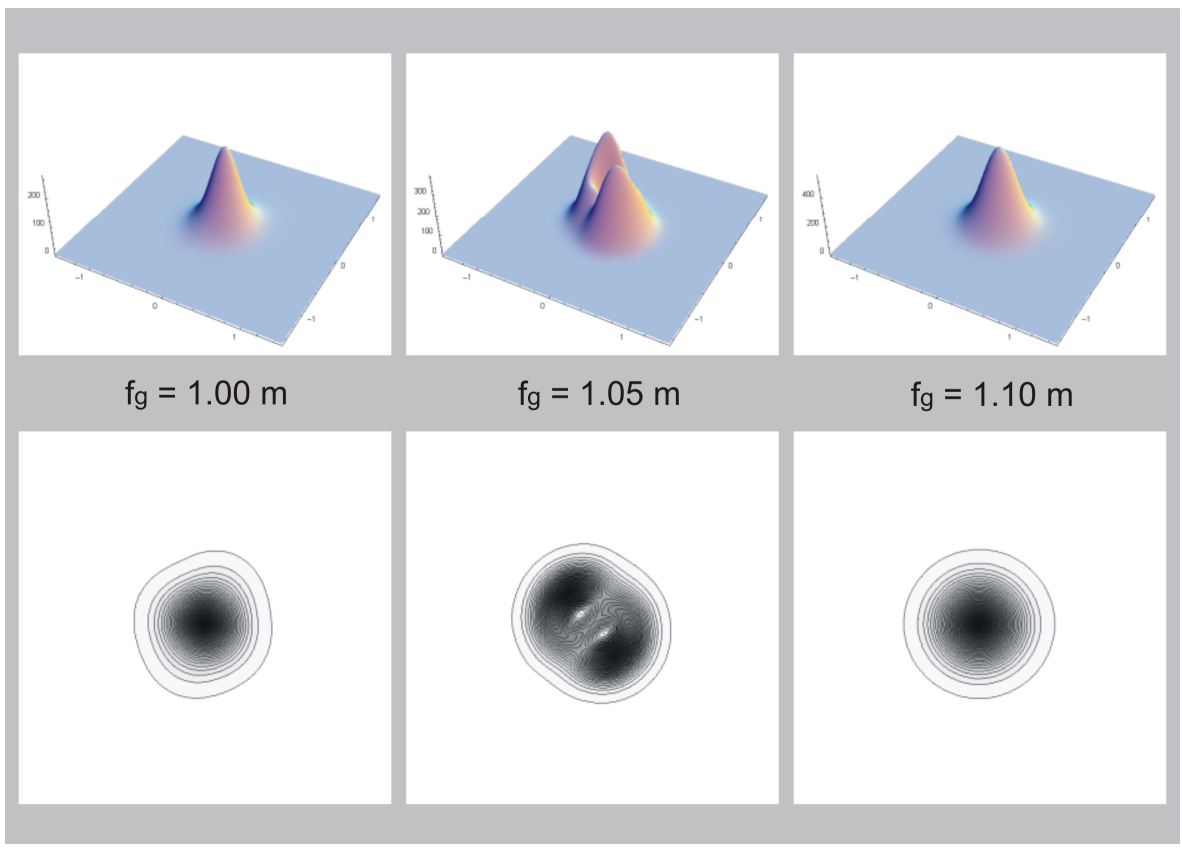

Fig. 16: Numerical simulations of the field distribution in the fundamental mode $\mathrm{V}$-shaped resonator as function of the focal length $f_{\mathrm{g}}$ of the disk. Left: near the threshold $\left(P_{\mathrm{p}}=250 \mathrm{~W}\right)$; middle: medium pump power $\left(P_{\mathrm{p}}=800 \mathrm{~W}\right)$; right: high pump power $\left(P_{\mathrm{p}}\right.$ $=1.8 \mathrm{~kW})$.

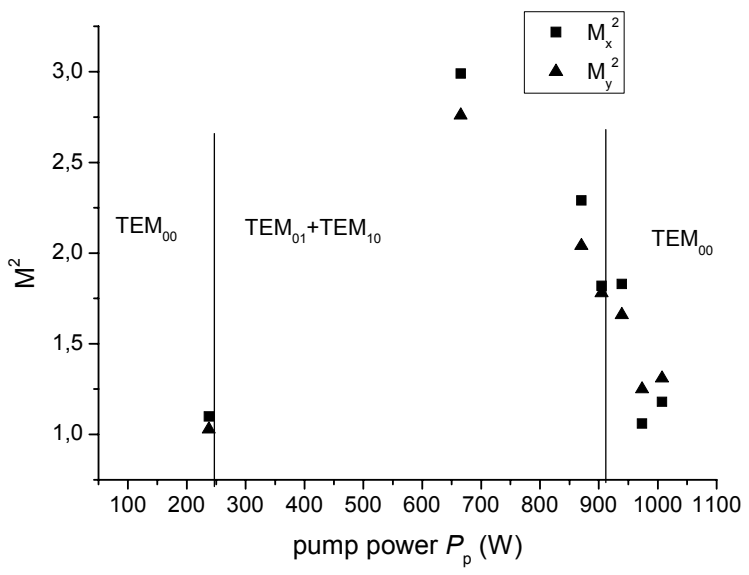

Fig. 17: Beam propagation factor $\mathrm{M}^{2}$ (x- and $\mathrm{y}$-direction) of the fundamental mode of the $\mathrm{V}$-shaped resonator as function of the pump power $P_{\mathrm{p}}$.

After further optimization ( $4 \%$ instead of $3 \%$ outcoupling transmission) the output power could be enhanced up to $360 \mathrm{~W}$ with an optical-optical efficiency of $33 \%$ and a beam quality of $\mathrm{M}^{2}=1.3$. 


\section{CONCLUSIONS}

The thin-disk laser concept is well suited to realize a high power solid state laser with fundamental mode emission. In our experiments, published fundamental mode laser powers could be exceeded. The experimental results are consistent with numerical simulations. Moreover, this concept offers the possibility of further optimizations considering pump power homogeneity and resonator design to enhance output power in the multi-kilowatt regime at high beam quality.

\section{REFERENCES}

R. Brockmann, K. Mann, "Disk lasers enable industrial manufacturing", LTJ, No. 3, June 2007.

Stolzenburg et. al., "Power scalable single frequency thin disk oscillator", Advanced Solid-State Photonics, the Optical Society of America, Washington, D.C. (2005).

3 K. Contag, M. Karszewski, C. Stewen, A. Giesen, H. Hügel, "Theoretical modelling and experimental investigations of the diode-pumped thin-disk Yb:YAG laser", Quantum Electronics 29 (8), 697 - 703 (1999).

4 V. Magni, "Multielement stable resonators containing a variable lens", JOSA A 4, Nr. 10, 1962 (1987). 